\title{
Specialty Grand Challenge: Sensor Networks
}

\author{
Guangjie Han* \\ Department of Information and Communication System, Hohai University, Changzhou, China
}

Keywords: sensor network, Internet of everything, edge computing, communication, information-physical world

\section{INTRODUCTION}

Nowadays, Sensors have been deployed all over the daily living environment, integrated into smart phones, smart watches, and other wireless terminal devices, and become the necessities in modern daily life. With the progress of IoT (Internet of Things) and AI (artificial intelligence), more wireless sensing devices will be used to extend human senses for providing accurate and comprehensive sensory data of life activities via networking. The International Data Corporation (IDC) reports that both the number of online devices and total generated data will reach unprecedented magnitudes in 2025. Their exponential increase heralds the advent of a new era named "Internet of Everything (IoE)". Obviously, mass data is an opportunity to develop data-driven technologies, but also a challenge for computational loading capacity. Given the transmission cost, information security, and system scalability, Sensor Network based schemes may be the current optimal solution (Akyildiz et al., 2002).

The field of Sensor Networks has gone through three major reforms ranging from version 1.0 (isolated static systems) to 3.0 (invisible adaptive, self-managing systems), each of which has witnessed the revolution of IoT technology.

In stage 1.0, i.e., Sensor Networks 1.0, the topics under continuous discussion are about sensor localization, intelligent management, interconnection, etc., on which most of current research products still concentrate. In other words, how to connect our physical world to the Internet through sensor networks is an everlasting hotspot. In moving forward, the fusion of sensors and networks must confront the following challenges:

Edited and reviewed by: Dermot Diamond

Dublin City University, Ireland

${ }^{*}$ Correspondence: Guangjie Han hanguangjie@gmail.com

Specialty section: This article was submitted to Soft Robotics,

a section of the journal Frontiers in Robotics and Al

Received: 27 April 2021 Accepted: 17 May 2021 Published: 01 June 2021

Citation: Han G (2021) Specialty Grand Challenge: Sensor Networks. Front. Sens. 2:700967. doi: 10.3389/fsens.2021.700967

\section{Challenge: Localization and Synchronization}

Non-line-of-sight propagation is a challenge in localization toward wireless sensor networks, especially in complex indoor environments. Besides, accurate target localization remains a challenge when switching between line-of-sight and non-line-of-sight environments. Traditional methods are more effective when there is line-of-sight between a node and one or a small number of neighboring beacon nodes. However, in an entirely non-line-of-sight environment, the traditional algorithm's performance decreases, and the computational complexity is very high (Mao et al., 2007). Traditional algorithms for identifying non-line-of-sight environments should not rely on the likelihood function, as the likelihood function is a fuzzy, soft decision method that can lead to unsatisfactory network boundary localization. When designing a time synchronization scheme for wireless sensor networks, the computational process and routing should not have too much complexity, considering that wireless sensor networks lack infrastructure, are distributed, have limited energy, and have limited storage and computing power (Rentel and Kunz, 2008). Many current time synchronization schemes for wireless sensor networks are based on linear rules for clock updates. However, in many practical situations, the algorithms for time synchronization may require a non-linear design. Therefore, we need to investigate non-linear average time synchronization schemes for wireless sensor networks and synchronization algorithms that take into account wireless channel fading and random delays. 


\section{Challenge: Protocols and Communication Systems}

The energy consumption of wireless transmission is proportional to the square or the fourth power of the communication distance. It is unreasonable for the sensor node to send its sensing data to the sink node directly. Thus, it is necessary to design proper protocols and communication systems to carry out data transmission in a multi-hop way. According to node transmission power, the ideal protocols are designed to minimize total communication distance or total jump in network data collection. Meanwhile, some key nodes inevitably required to relay node forwarding data, leading to depletion of their power and formation of an energy hole in the multi-hops transmission scenes. To address the aforementioned problems, many current works aim to design the protocols and communication systems for improving channel capacity, network scalability, and rebalancing energy consumption. However, there still exist challenges in the design and deployment of the actual scenes.

\section{Challenge: Energy Optimization for Sensor Networks}

The introduction of sensor node energy charging and conservation mechanisms can effectively reduce the amount of communication between sensor nodes, thus extending the nodes' dormancy time. Traditional methods have to a certain extent alleviated the problem of insufficient sensor node energy and improved sensor networks' operational performance in specific application scenarios (He et al., 2006). However, these schemes have not been well studied in terms of fast node discovery, coordination of heterogeneous devices, and trade-offs between energy efficiency and data latency. In particular, with the proposed and rapid popularity of cross-layer joint hibernation mechanisms, there is no mature solutions on how to apply sensor node energy charging and saving mechanisms in sensor networks that have already adopted cross-layer joint hibernation mechanisms. Therefore, the sensor node energy charging and saving mechanism still need to be further investigated.

For stage 2.0, the rapid development of network virtualization prompts the sensor network be more scalable and can be defined by software, i.e., software-defined. By software-defined technologies, the network functions can be extracted from the underlying data transfer infrastructure, leading to a uniform network control plane. This is the so-called network function virtualization or Software-Defined Networking. The network virtualization technique enables machine-level real-time functionality in security prevention, control systems, and other traditional operational functions. For example, self-organization, security, secrecy, and privacy, etc.

\section{Challenge: Security, Secrecy, and Privacy}

Threats to security, secrecy, and privacy are huge for sensor networks, maybe caused by social control. While connecting into the Internet, all the online devices have to deal with the same problems of the current networks due to the similar underlying technologies. Besides, either for the data senders or receivers, the data need to be adequately protected to avoid privacy disclosure and data modification from passive and active attacks in transit. Especially for the future IoE era, the Internet-oriented sensor networks emphasize the autonomous interactions among the objects (sensors), making them "smart", but also besiege them with attackers (Sikder et al., 2021). Thus, the security, secrecy, and privacy mechanisms in sensor networks still need to be further investigated and strengthened.

\section{Challenge: Self-Organization/ Software-Defined Networking}

The rapid development of Software-Defined Networking (SDN) or Software-Defined Everything (SDE) technologies promotes the re-definition of self-organization in sensor networks, especially at the control aspect of the networking self-organization. Instead of networking self-organization in a distributed manner, the SDN technologies break this rule and decouples the networking control plane from the networking data delivery plane, leading to a united network control plane, i.e., the SDN controller (Kirkpatrick, 2013). Through the SDN controller, an abstract view about the networking states can be acquired, and the operations for networking organization can be intensively determined and deployed (Kreutz et al., 2014). The SDN-enabled networking self-organization can provide scalable networking control for the sensor networks, e.g., networking mobility management (Bi et al., 2019; Lin et al., 2020), cross-domain services support, etc., and have attracted intense research interest from both industry and academia. However, although the SDN technologies innovate the networking self-organization services, many challenging issues still have to be confronted and addressed, e.g., how to deploy and determine the SDN controller, how to guarantee the safety of the network control tunnel (Scott-Hayward, 2015), how to define the wireless control interfaces for deploying the self-organization operations, etc.

Different from the aforementioned two stages, in Sensor Networks 3.0, the wave of artificial intelligence (AI) enables the sensor networks be with the abilities of self-awareness including self-managing, self-healing, self-collection, etc. Expectantly, AI will reduce the coupling degree of the sensor networks' control component, meanwhile, improve the data collection efficiency of the sensor networks. Some typical applications include edge computing for various types of heterogeneous sensor networks, cooperative mechanism implementations in sensor networks, etc.

\section{Challenge: Cooperative Mechanism in Sensor Networks}

In WSNs, It is not recommended that nodes work independently due to their finite energy. Thus, most tasks of WSNs are completed via cooperative mechanisms. On the one hand, many environment-aware functions require data collaboration among multiple nodes. On the other hand, the collected data from the sensing node usually needs to be first sent to the sink node under cooperative control mechanisms instead of directly linking it to the remote control center. For the management of 
large-scale networks, clustering is the first step of cooperative mechanism, e.g., the clustering schemes based on path information and destination in IoV. There is no doubt that its rationality fundamentally determines the effectiveness of the subsequent operations. Furthermore, how to achieve virtualization management of node resources, design task allocation strategy, and summarize sensing and calculation results are also the non-trivial tasks.

\section{Challenge: Edge Computing for Scalable Networks}

Due to the nature of WSNs, the density nodes need simultaneous connectivity. System scalability has become a significant feature of WSNs for edge computing. A sensor network has two types of scalability operations: vertical and horizontal scalability, which are employed to add or remove computing resource nodes and sensing nodes, respectively. Scalable networks bring agile computing resources but are inundated with uncertainty. At present, edge computing has attracted continuous attention, and it performs better in many applications (Shi et al., 2016). However, many problems still need to be investigated in practical applications, e.g., computational performance optimization, security, interoperability, and intelligent service management.

\section{Challenge: Practical Implementations in loT}

With the technological strides of IoT and AI (Artificial Intelligence), novel sensing modes and methods are integrated into WSNs successively, such as multi-functional active sensors, smart/intelligent sensors, edge computing nodes, etc. The application fields of WSNs have also been extended into smart home/traffic/city, healthcare, and resource exploration and management, etc.

\section{Wireless Body Area Networks}

WBANs-based applications have been widely employed in military, entertainment, consumer electronics, smart home, public services, healthcare, and other fields. With different functional sensors, WBANs provide a novel model for human health monitoring, which has great significance and demand in disease monitoring, health recovery, special population monitoring, etc. Most of the existing modeling methods for WBANs are always considering the human body as a whole objective (Movassaghi et al., 2014). A series of sensors with different functions are integrated as a data collector or feature extractor at the front of a classification framework and output at least one signal to feed the classifiers for monitoring the physiological status. Also, many researchers formulate sensing node distribution as a network-based skeleton to meet both movement rules of the human body and the mobile network's performance in some scenes of motion/ behavior analysis (Roy et al., 2020). However, despite these efforts, challenges still remain due to the diversity of the WBAN-based applications as application scenes have significantly different demands on network performance, which need to be further investigated.

\section{Internet of Vehicles}

The Internet of Vehicles (IoV) is a specialized IoT application, in which decentralized networks of vehicles and nodes strive to achieve common goals under cooperative mechanisms. IoV allows vehicles to exchange information with other vehicles or transport infrastructures, denoted as vehicle-to-vehicle communications $(\mathrm{V} 2 \mathrm{~V})$ and vehicle-to-infrastructure communications (V2I), respectively. Given its importance in future smart cities, lots of applications, such as autonomous driving, route planning, mobile sensing, accident warning systems, traffic analysis, intelligent transportation systems, IoV has captured the interests of the research communities (Wang et al., 2020). Although some solutions for the aforementioned applications have been proposed, there still exist many issues far to be solved.

\section{Underwater and Underground Sensor Networks}

The major breakthroughs and advances in underwater/ underground information technology (e.g., underwater/ underground sensing technology, underwater/underground sensor localization, etc.), underwater/underground robotics (e.g., underwater/underground robot, underwater/ underground sensor, underwater autonomous underwater vehicles, etc.), etc., give rise to the concept of Internet of Underwater/Underground Things (Jing et al., 2020). The Internet of Underwater/Underground Things can be expressed in many forms. Underwater/underground sensor networks have been proposed as the most effective frameworks to survey the ocean or monitor underground activities or resources since they can provide scalable data collection by particular communication technologies, e.g., underwater acoustic-based communication. However, due to the hostile communication environment, underwater/ underground sensor networks face challenges and open research issues. For instance, underwater/underground ground communication is a topic that never ends and is progressing slowly. Meanwhile, current technologies for localizing underwater/underground sensors still have many limitations. Furthermore, routing or data transfer scheduling in these particular environments is also a hot research issue.

The Sensor Networks transformation journey has already begun. We can't go straight from 1.0 to 3.0 , we have to build the capabilities needed at each stage to move forward one stage at a time. But the result will be a Sensor Networks transformation that creates a modern, flexible, efficient, and resilient information-physical world.

\section{AUTHOR CONTRIBUTIONS}

The author confirms being the sole contributor of this work and has approved it for publication. 


\section{REFERENCES}

Akyildiz, I. F., Weilian Su, W., Sankarasubramaniam, Y., and Cayirci, E. (2002). A Survey on Sensor Networks. IEEE Commun. Mag. 40 (8), 102-114. doi:10.1109/ mcom.2002.1024422

Bi, Y., Han, G., Lin, C., Guizani, M., and Wang, X. (2019). Mobility Management for Intro/Inter Domain Handover in Software-Defined Networks. IEEE J. Select. Areas Commun. 37 (8), 1739-1754. doi:10.1109/jsac.2019.2927097

He, T., Krishnamurthy, S., Luo, L., Yan, T., Gu, L., Stoleru, R., et al. (2006). VigilNet. ACM Trans. Sen. Netw. 2 (1), 1-38. doi:10.1145/1138127. 1138128

Jing, S., Hall, J., Zheng, Y. R., and Xiao, C. (2020). Signal Detection for Underwater IoT Devices with Long and Sparse Channels. IEEE Internet Things J. 7 (8), 6664-6675. doi:10.1109/JIOT.2020.2984532

Kirkpatrick, K. (2013). Software-defined Networking. Commun. ACM 56 (9), 16-19. doi:10.1145/2500468.2500473

Kreutz, D., Ramos, F. M. V., Verissimo, P. E., Rothenberg, C. E., Azodolmolky, S., and Uhlig, S. (2014). Software-defined Networking: A Comprehensive Survey [J]. Proc. IEEE 103 (1), 14-76. doi:10.1109/JPROC.2014.2371999

Lin, C., Han, G., Qi, X., Guizani, M., and Shu, L. (2020). A Distributed Mobile Fog Computing Scheme for Mobile Delay-Sensitive Applications in SDN-Enabled Vehicular Networks. IEEE Trans. Veh. Technol. 69 (5), 5481-5493. doi:10.1109/ tvt.2020.2980934

Mao, G., Fidan, B., and Anderson, B. D. (2007). Wireless Sensor Network Localization Techniques. Computer networks 51 (10), 2529-2553. doi:10. 1016/j.comnet.2006.11.018

Movassaghi, S., Abolhasan, M., Lipman, J., Smith, D., and Jamalipour, A. (2014). Wireless Body Area Networks: A Survey. IEEE Commun. Surv. Tutorials 16 (3), 1658-1686. doi:10.1109/surv.2013.121313.00064
Rentel, C. H., and Kunz, T. (2008). A Mutual Network Synchronization Method for Wireless Ad Hoc and Sensor Networks. IEEE Trans. Mobile Comput. 7 (5), 633-646. doi:10.1109/tmc.2007.70787

Roy, A., et al. (2020). "Activity-Aware Data Rate Tuning in Wireless Body Area Networks[C]," in GLOBECOM 2020 - 2020 IEEE Global Communications Conference, Taipei, Taiwan, 01-06. 7-11 December 2020, . IEEE.

Scott-Hayward, S. (2015). "Design and Deployment of Secure, Robust, and Resilient SDN Controllers[C]," in Proceedings of the 2015 1st IEEE conference on network Softwarization (NetSoft) (IEEE), 13-17 April 2015, London, UK: IEEE, 1-5.

Shi, W., Cao, J., Zhang, Q., Li, Y., and Xu, L. (2016). Edge Computing: Vision and Challenges. IEEE Internet Things J. 3 (5), 637-646. doi:10.1109/jiot.2016.2579198

Sikder, A. K., Petracca, G., Aksu, H., Jaeger, T., and Uluagac, A. S. (2021). A Survey on Sensor-Based Threats and Attacks to Smart Devices and Applications[J]. IEEE Commun. Surv. Tutorials. 23 (2), 1125-1159. doi:10.1109/COMST.2021.3064507

Wang, H., Liu, T., Kim, B. G., Lin, C. W., Shiraishi, S., Xie, J., and Han, Z. (2020). Architectural Design Alternatives Based on Cloud/Edge/Fog Computing for Connected Vehicles[J]. IEEE Commun. Surv. Tutorials. 22 (4), 2349-2377. doi:10.1109/COMST.2020.3020854

Conflict of Interest: The author declares that the research was conducted in the absence of any commercial or financial relationships that could be construed as a potential conflict of interest.

Copyright (๑) 2021 Han. This is an open-access article distributed under the terms of the Creative Commons Attribution License (CC BY). The use, distribution or reproduction in other forums is permitted, provided the original author(s) and the copyright owner(s) are credited and that the original publication in this journal is cited, in accordance with accepted academic practice. No use, distribution or reproduction is permitted which does not comply with these terms. 